\title{
THz radiation of stabilized dense electron bunches
}

\author{
A.V. Savilov $^{1}$, I.V. Bandurkin ${ }^{1}$, I.S. Kurakin ${ }^{1}$, Yu.S. Oparina ${ }^{1}$, \\ V.L. Bratman ${ }^{1,2}$, N. Balal ${ }^{2}$, Yu. Lurie ${ }^{2}$ \\ ${ }^{1}$ Institute of Applied Physics RAS, N. Novgorod, Russia, savilov@appl.sci-nnov.ru \\ ${ }^{2}$ Ariel University, Ariel, Israel
}

Laser-driven photo-injectors are capable to form very compact and dense electron bunches with a particles energy of 3-6 MeV, picosecond and subpicosecond duration, and charge of the order of 1 nC. When moving in the periodic field of the undulator with a period of a few centimeters, such bunches can generate coherent radiation in the nearterahertz range. The power and duration of such a generation is limited due to an increase in the axial length of the electron bunch under the action of the Coulomb repulsion, which under normal conditions does not allow for the effective implementation of such a scheme for dense $\mathrm{nC}$ bunches [1]. Therefore, a special methods of stabilization of the axial length of the operating bunch during its motion over a long enough electron-wave interaction region should be used. The report describes two such methods, namely, the so-called "negative-mass" stabilization and the radiative compression of electron bunches.

\section{Negative-mass stabilization and compression}

The negative-mass regime of the electron motion is realized in a combination of periodic undulator field and relatively strong homogeneous axial magnetic field (Fig. 1 a). The cyclotron frequency corresponding to the axial field should be slightly higher than the undulator bounce-frequency of the particle. In this case, the Coulomb field inside the bunch leads not to repulsion of electrons but to their mutual attraction [1]. This effect is a result of an abnormal dependence of the velocity of undulator oscillations of electrons on the cyclotron frequency (Fig. 1 b). Let us consider a particle moving in the front of the bunch (Fig. 1 c). The Coulomb field accelerates this electron, and its relativistic cyclotron frequency decreases. The electron approaches the undulator-cyclotron resonance, which is accompanied by a resonant increase in its transverse velocity; this leads to decrease in the axial electron velocity.

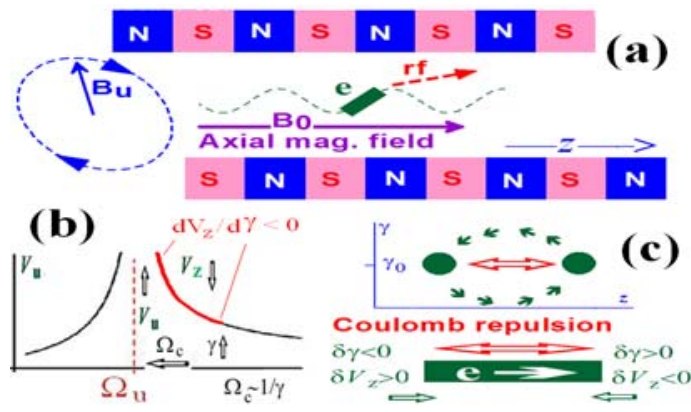

Fig. 1. (a): Electron motion in the combined helical undulator and uniformaxial fields. (b) Characteristic dependence of the transverse electron velocity on the cyclotron frequency. (c) Coulomb attraction and oscillations of electrons in the "negative-mass" regime.

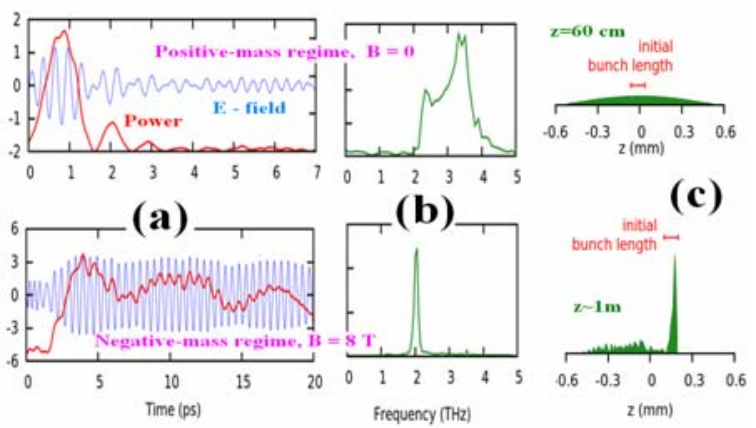

Fig. 2. (a): Radiated wave field and power (red) in the "positive-mass" and "negative-mass" regimes. (b): Spectra of the radiated rf signal. (c): Axial distributions of the charge in the bunch after the $60 \mathrm{~cm}$ trip in the positive-mass regime and after the $1 \mathrm{~m}$ trip in the negative-mass regime.

Thus, increasing the energy of the particle causes it to slow down in the longitudinal direction. The opposite situation takes place for the particle moving in the tail of the bunch; this particle is decelerated by the coulomb filed, and this leads to an increase in the axial velocity. Overall, the Coulomb interaction of particles leads to compression of the bunch in the longitudinal direction. Such a behavior of electrons can be regarded as a consequence of its effective mass being negative [2,3], and this is very similar to the negativemass effect in cyclotron masers [4-7].

Numerical simulations show $[1,8]$ that in a certain range of parameters, significant improve of the radiation characteristics is possible when using the described scheme. Figure 2 illustrates an example, where an electron bunch with the particles energy of $5.5 \mathrm{MeV}$ scatters the undulator field having a period of $2.5 \mathrm{~cm}$ into the wave with a frequency of about 2 THz. In this case, the resonant cyclotron frequency corresponds to the axial field of about $5 \mathrm{~T}$, and effective negative mass regime is realized at magnetic fields near $8 \mathrm{~T}$ and the undulator field amplitude of about $0.2 \mathrm{~T}$ (Fig. 2). Simulations predict generation of a 20 ps rf pulse with a power of $\sim 10 \mathrm{MW}$. According to simulations [8], the negative-mass regime can provide axial stabilization of extremely dense (with charges up to several $\mathrm{nC}$ ) picosecond bunches. A prototype of the negative-mass undulator with a $8 \mathrm{~T}$ axial magnetic field is designed and tested in the experiment [9]. There is also an interesting option to use this regime for the axial compression of dense bunches by their own Coulomb fields, when the undulator is used not for the radiation but for providing the negativemass electron motion only [10]. Since in this situation there is no goal to achieve $\mathrm{THz}$ radiation, undulators with longer periods can be used, and a lower magnitudes of the axial magnetic field are required to provide the negative-mass electron motion. 


\section{Radiative compression}

An alternative compression method can be the undulator super-radiative radiation of a longwavelength wave in an auxiliary long-period undulator [11]. A relatively long electron bunch propagates in a simple (with no axial magnetic field) helical undulator inside a waveguide (Fig. 3 a) and radiates a short wave packet propagating with a group velocity equal to the electron axial velocity (Fig. $3 \mathrm{~b}$ ). Since the wavelength of the radiated wave is longer than the bunch length, the emission has the spontaneous coherent character. Such emission results in axial compression of the bunch (Fig. 3 c). This is due to the fact that in the situation described above the phase of the radiated wave is correlated in a certain way with the electron bunch phase, namely, there is a $\pi / 4$ shift between the bunch center and maximum of the decelerating wave phase (Fig. $3 \mathrm{~d}$ ). In the case of a bunch being four times shorter than the wavelength, the bunch is compressed in the radiated field, because the front of the bunch is placed in the maximum of the decelerating wave phase whereas the tail is placed close to the "zero" wave field.

This effect can be used for creation of a "bicolor" $\mathrm{THz}$ source based on the spontaneous emission from a short bunch, so that the super-radiation of the auxiliary long-wavelength wave is used to compress the bunch down to a size shorter than the wavelength of the short-wavelength wave. Figure 4 illustrates an example, where a $0.1 \mathrm{nC} 3 \mathrm{MeV} 0.6 \mathrm{~mm}$ bunch moves in two undulators $\left(\lambda_{u, 1}=2.3 \mathrm{~cm} \quad\right.$ and $\left.\lambda_{u, 2}=1.1 \mathrm{~cm}\right)$. At the first stage, electron oscillations in the first undulator lead to the spontaneous coherent radiation of the long-wavelength $\left(\lambda_{1}=1.2 \mathrm{~mm}\right)$ compressing wave. During this process, the bunch is compressed several times. This makes possible the spontaneous coherent radiation of the short-wavelength $\left(\lambda_{2}=0.3 \mathrm{~mm}\right)$ wave in the second undulator with a relatively high ( $\sim 10 \%)$ electron efficiency.

The work is supported by IAP RAS Project 00352014-0012, Russian Foundation for Basic Research (Project 18-32-00351 and 16-02-00794), and the Israeli Ministry of Science, Technology and Space.
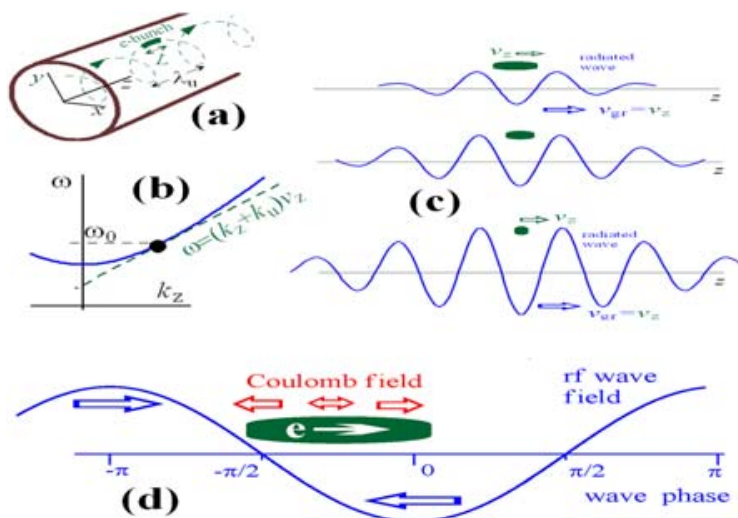

Fig. 3. (a): Electron bunch moving along a helical undulator. (b): Dispersion diagram of the operating waveguide mode. (c) Super-radiative radiation and the bunch compression. (d): Bunch phase with respect to the wave.

\section{References}

1. Balal, N., Bandurkin, I. V., Bratman, V. L., Magory, E., Savilov, A. V. Negative-mass mitigation of Coulomb repulsion for terahertz undulator radiation of electron bunches // Appl. Phys. Lett. 2015. V. 107. P. 163505.

2. Freund, H. P., Sprangle, $P$. Unstable electrostatic beam modes in free electron-laser systems // Phys. Rev. A. 1983. V. 28. P. 1835

3. Ginzburg, N. S., Peskov, N. Yu. Non-linear theory of relativistic ubitrons with electron beams formed in an adiabatically increasing undulator field and a uniform longitudinal magnetic field // Tech. Phys. 1988. V. 58. P. 859.

4. Bratman, V. L. Instability of orbital motion in a layer of electrons rotating in a uniform magnetic field // Tech. Phys. 1976. V. 46. P. 2030.

5. Bondeson, A., Antonsen, T. Space-charge instabilities in gyrotron beams // Int. J. Electron. 1986. V. 61. P. 855.

6. Bratman, V. L., Savilov, A. V. Phase mixing of bunches and decrease of negative-mass instability increments in cyclotron resonance masers // Phys. Plasmas. 1995. V. 2. P. 557.

7. Savilov, A. V. Negative-mass instability in magnetron-injection guns // Phys. Plasmas. 1997. V. 4. P. 2276.

8. Lurie, Yu., Bratman, V. L., Savilov, A. V. Energy enhancement and spectrum narrowing in terahertz electron sources due to negative mass instability // Phys. Rev. AB. 2016. V. 19. P. 050704.

9. Balal, N., Bandurkin, I.V., Bratman, V.L., Fedotov, A.E. Helical undulator based on redistribution of uniform magnetic field // Phys. Rev. AB. 2017. V. 20. P. 122401.

10. Bandurkin, I.V., Kurakin, I.S., Savilov, A. V. Compression of a photoinjector electron bunch in the negativemass undulator // Phys. Rev. AB. 2017. V. 20. P. 020704.

11. Bandurkin, I.V., Oparina, Y.S., Savilov, A.V. Superradiative self-compression of photo-injector electron bunches // Appl. Phys. Lett. 2017. V. 110. P. 263508.

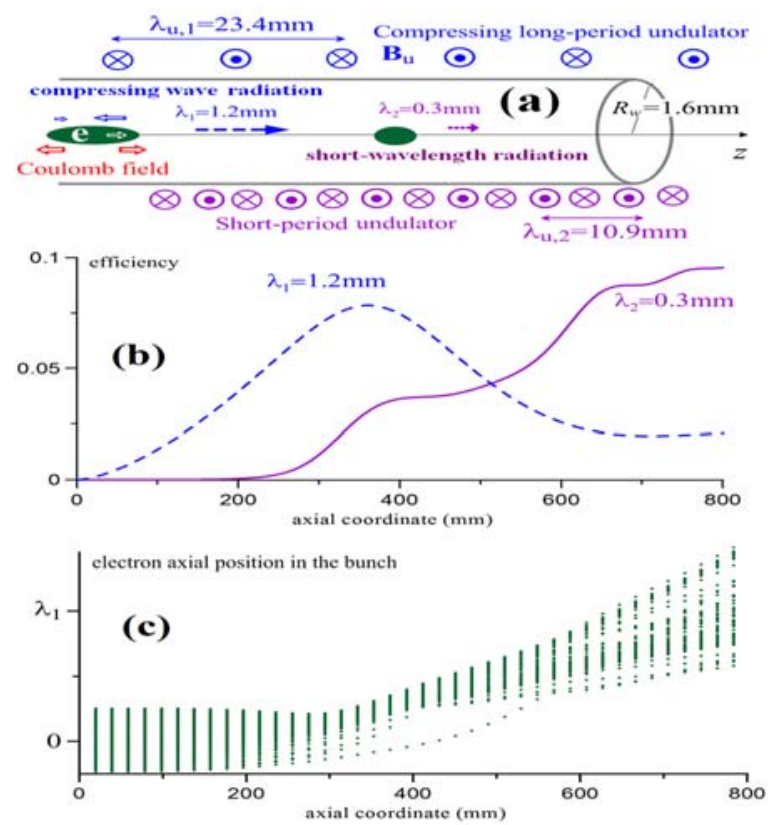

Fig. 4. (a): "Bicolor" THz source based on the spontaneous emission of two waves. (b): Efficiency of radiation of the two waves versus the axial coordinate of the bunch. (c): Evolution of axial distribution of electrons inside the bunch. 